\title{
Newly designed hemagglutinin-Der p 2 chimera is a potential candidate for allergen specific immunotherapy
}

\author{
Ivan Mrkića, Rajna Minić ${ }^{\mathrm{b}}$, Dragan Popovićc , Irena Živkovićb ${ }^{\mathrm{b}}$, Marija Gavrović-Jankulovićc ${ }^{\mathrm{d}, *}$ \\ ${ }^{a}$ Innovation Center, University of Belgrade - Faculty of Chemistry, Belgrade, Serbia \\ ${ }^{\mathrm{b}}$ Institute of Virology, Vaccines and Sera, Torlak, Belgrade, Serbia \\ ${ }^{\mathrm{c}}$ Department of Chemistry - IChTM, University of Belgrade, Belgrade, Serbia \\ d Department of Biochemistry, University of Belgrade Faculty of Chemistry, Belgrade, Serbia
}

\section{A R T I C L E I N F O}

\section{Keywords:}

Allergy

House dust mites

Vaccine

Immunotherapy

\begin{abstract}
A B S T R A C T
Aim

To investigate the immunomodulatory potential of a chimera composed of the receptor-binding domain of hemagglutinin 1 (H1s) from Influenza virus and Der p 2 (D2) allergen for allergen-specific immunotherapy of house-dust mite allergy (HDM).

Main methods: H1sD2 chimera and D2 allergen were produced by genetic engineering in E. coli. Recombinant antigens were extracted from inclusion bodies by urea, then refolded and purified by immobilized- metal affinity chromatography (IMAC). Purity was verified by 2D-PAGE and secondary structures were assessed by CD spectroscopy. IgE reactivity of $\mathrm{H} 1 \mathrm{sD} 2$ and D2 was tested in western blot with sera from 8 persons with clinical history of HDM allergy. Immunogenicity of H1sD2 and D2 were analyzed in Balb/c mice. Cytokine profile was analyzed by ELISA after stimulation of mouse spleen cells with H1sD2 and D2. Leukocyte population abundance of cells isolated from spleen and lymph node was assessed by flow cytometry.

Key findings: Purified recombinant proteins H1sD2 (42 kDa) and D2 (15 kDa) revealed well defined secondary structures, and preserved IgE reactive epitopes. Analysis of supernatants of mouse spleen cells after stimulation with $\mathrm{H} 1 \mathrm{sD} 2$ and $\mathrm{D} 2$, revealed a qualitatively different cytokine profile from H1sD2 immunized mouse cells (increase in IL10). CD8 + cells were decreased in the lymph node of D2 immunized mice, whereas H1sD2 immunization led to an increase of CD8 + cells in both the lymph node and the spleen.

Significance: H1sD2 chimera attenuates Der p 2-inherent Th2 response and directs the immune response toward Th1 and Treg phenotype.
\end{abstract}

\section{Introduction}

Allergy represents a chronic non-communicable disease with increasing prevalence worldwide (approximately $30 \%$ to $40 \%$ of the world population) [1,2]. It is an IgE mediated immune disorder characterized by Th2 phenotype which develops against innocuous environmental antigens in genetically predisposed (atopic) persons. Clinical manifestations of an allergic response are triggered when allergen binds and cross-links specific IgE antibodies anchored for high affinity IgE receptor (FceRI) on the effector cells (mast cells and basophils) which then release biologically active mediators (histamine, leukotrienes, prostaglandins, cytokines). The only therapeutic option with curative effect in allergic patients is allergen-specific immunotherapy (AIT). AIT was introduced by Noon [3] more than a century ago and it is still the only disease-modifying treatment in allergy. AIT induces reduction of clinical symptoms and prevents the development of asthma and new allergen sensitizations, by the introduction of defined amounts of allergen to an allergic patient for a certain period of time.

AIT is predominantly performed with allergen extracts which besides disease-causing allergens contain nonallergenic components with adjuvant activity that can drive the immune response via a Th2 pathway [4,5] and consequently can contribute to elicitation of IgE sensitization to other allergens present in the extract. In addition, variations in content and amounts of individual allergens in allergen extracts can compromise efficacy of AIT. To improve safety and efficacy of the therapeutic approach, replacement of allergen extracts with defined amounts of recombinant allergens has been proposed as an alternative [6]. Good clinical efficacy was reported in clinical trials conducted with recombinant allergen preparations (timothy grass pollen, birch pollen)

\footnotetext{
* Corresponding author.

E-mail address: mgavrov@chem.bg.ac.rs (M. Gavrović-Jankulović).
} 
in comparison to placebo. Therapeutic treatment is mainly associated with modification of the specific immune response in terms of promotion of specific IgG4 and reduction of specific IgE antibodies, together with the induction of IL-10-producing regulatory T cells [7].

However, in spite of beneficial clinical efficacy, recombinant allergen preparations revealed poor immunogenicity, and therefore they do not induce significantly better therapeutic effects than natural extracts $[8,9]$. Moreover, there is a need for employment of novel immunomodulators capable to enhance the innate immune response in order to economically justify the production of recombinant allergy vaccines [10].

House dust mite is a well-known cause of respiratory allergic disease worldwide. Also, diagnosis and treatment is still a challenge [11]. AIT in house dust mite allergy has been shown to correct the underlying allergic cause safely and effectively, with long-term effect after the end of treatment $[12,13]$.

According to their molecular properties mite allergens are classified in 24 groups, but almost $80-90 \%$ of mite-allergic persons are sensitized to Der p 1 and Der p 2 [14]. While biologically active Der p 1 (cysteine protease) can compromise barrier of the bronchial epithelium, allowing allergens and nonallergic components to reach cells of the immune system, Der p 2 has an immunoglobulin-like tertiary structure around a hydrophobic cavity [15] which can have an intrinsic adjuvant effect due to its proposed LPS binding abilities and contributes to the allergenicity of such molecules [16]. It has been found that Der p 2 reveals structural homology with MD-2 and shows auto-adjuvant properties which contributes to allergic sensitization [16].

Influenza is one of the major infectious diseases of the human population. Viral infections, including the influenza A virus infection, generally produce a cytokine milieu that favors the development of type 1 immunity [17]. Influenza vaccines provide the most effective control of seasonal outbreaks [18]. The key protective antigen in natural immunity and vaccines of Influenza virus is the envelope-associated hemagglutinin (HA) [19]. It is the surface glycoprotein inserted into the lipid bilayer as spike, which promotes virus entry into the cells. The HA is a trimeric glycoprotein made of a globular receptor-binding domain (RBD) settled onto a stem domain [20].

Modulation of the immune response toward Th1 profile in the context of house dust mite allergy has already been explored. For instance, bacilli Calmette-Guerin (rBCG), a vaccine strain against tuberculosis as a strong inducer of Th1 response had been used as a delivery vehicle for the presentation of foreign antigens to the immune system. Inoculation of recombinant $\mathrm{rBCG}$ with Der $\mathrm{p} 2$ cause a Th1-type immune response that corrects Der p 2 induced allergic sensitization and consequent development of airway inflammation [21].

In this study immunodominant epitope of HA1 was exploited as modulator for reshaping inherent Th2 response of Der $\mathrm{p} 2$. In a previous study we have showed that a chimera composed of the native sequence of the receptor-binding domain and a part of stalk segment of HA1 heamagglutinin (HA63-351) and Der p 2 revealed immunomodulatory potential in a mouse model (Balb/c) [22]. By intranasal application of H1D2, reduced levels of D2 specific IgE and increased D2 specific IgG and IgA were detected. In addition, a higher level of effectors CD4 + CD25 + spleen lymphocytes were also observed in a group of mice which received i.n. instillations of H1D2.

In this study we redesigned H1D2 chimera to obtain better solubility by excluding the stalk domain and coupling only the receptor-binding domain of $\mathrm{H} 1$ with Der p 2. Immunogenicity of the novel chimera $\mathrm{H} 1 \mathrm{sD} 2$ produced by the recombinant DNA technology was tested in Balb/c mice.

\section{Materials and methods}

\subsection{Design of H1sD2 chimera}

A model of the H1sD2 chimera was designed form the crystal structure of a 2009 H1N1 influenza virus hemagglutinin, PDB entry 4M4Y [23] (chain A from amino acid residue 54 to 276 with original PDB residue numbering is used), and structure of the major house dust mite allergen Der p 2, PDB entry 1A9V [24] (model 1 from the NMR structure is used), in a similar way as it was previously described [25], but with the following changes. The original H1D2 construct was shortened for 60 amino acids (part of stalk domain) from the $\mathrm{C}$ terminus of $\mathrm{H} 1$ hemagglutinin. The resulting H1sD2 is composed of 371 amino acids which encodes for the HA receptor-binding domain (HA63-291), linker PGPG, and Der p 2 with the 6 His tag on $C$ terminus.

A proline-glycine-proline-glycine (PGPG) hinge is created between $\mathrm{H} 1 \mathrm{~s}$ and D2 to prevent steric hindrance, while a $6 \mathrm{His}$ tag is added at the C-terminus of the D2 protein for the purification purpose. The PGPG linker is modeled as a $\beta$-turn and the $6 \mathrm{His}$ tag as a $\beta$-strand. The computer-generated H1sD2 chimeric structure is obtained by connecting the four segments and adding the hydrogen atoms in Discovery Studio [26] followed by a full structural minimization in NAMD 2.9 [27] with CHARMM22 force field in implicit solvent/water model for $50 \mathrm{ps}$. The structure obtained this way is relaxed and strain-free without any bond and torsion angle constrains and van der Waals clashes. The short linker part efficiently prevents the non-bonded interactions between the two main domains, H1s and D2, by keeping them distant. Therefore, their tertiary structures and binding sites remain intact and available for interactions with the immune system. The structure of the $6 \mathrm{His}$ tag already possesses His residues in a favorable orientation such that can easily get coordinated to $\mathrm{Co}^{2+}$ cations. Final structure of the H1sD2 chimera is shown in Fig. 1A, and the amino acid sequence is presented in Fig. 1B. Depiction is made in Discovery Studio [26].

\subsection{Strains and plasmids}

Escherichia coli strains DH5a (Invitrogen Thermo Fisher Scientific, Carlsbad, USA) and BL21-CodonPlus (DE3)-RIPL (Agilent Technologies Inc., La Jolla, USA) were employed as host either for propagation of plasmid pET23b (Merck Millipore, Merck KGaA, Darmstadt, Germany) or for expression of recombinant D2 and H1sD2 proteins, respectively. Bacteria were grown in LB medium supplemented with antibiotics.

\subsection{Expression, purification and refolding of the recombinant antigens}

pET23b vector was used for expression of Der p 2 and H1sD2 genes.

A

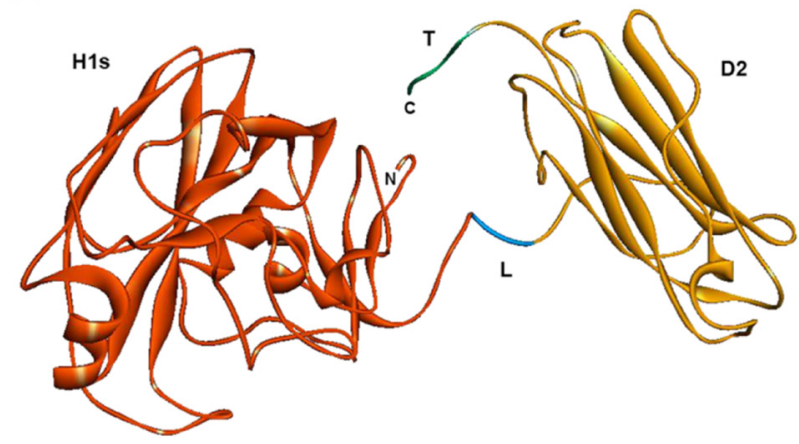

B

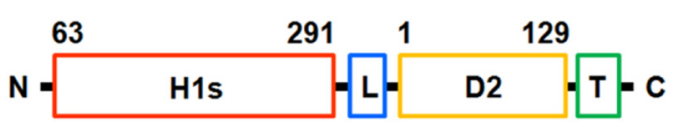

Fig. 1. Design of H1sD2 chimera: (A) modeled structure of H1sD2; (B) schematic illustration of the creation of H1sD2. H1s segment is shown in orange, Der p 2 in yellow, PGPG linker in blue and the 6His tag in green color. (For interpretation of the references to color in this figure legend, the reader is referred to the web version of this article.) 
Recombinant protein was produced in BL21-CodonPlus (DE3)-RIPL cells as previously described [22].

Gene coding for H1D2 [22] was used as a template for creation of H1sD2 construct. Briefly, DNA sequence of H1s and of Der p 2 were amplified by PCR with the following primers: H1s for 5'-CTAGCTAGC ATGGGTGTTGCCCC-3', H1s rev 5'-ACGCGTCGACTGTATTCATATCGT GAAC- $3^{\prime}$ with restriction sites for Nhe $I$ and Sal I; Der p 2 for $5^{\prime}$-ACGC GTCGACCCAGGTCCGGGTG-3', Der p 2 rev 5'-CCGCTCGAGATCACGG ATTTTAGCATGAG-3' with restriction sites for Sal $I$ and Xho $I$. The cloning strategy introduced $6 \mathrm{His}$ tag to the $\mathrm{C}$ terminus. After cut with respective restriction enzymes both PCR products were ligated into pET23b resulting in the generation of H1sD2 construct. OFR was confirmed by DNA sequencing.

Expression of H1sD2 gene has been done in BL21-CodonPlus (DE3)RIPL cells in LB medium $\left(10 \mathrm{~g} \mathrm{~L}^{-1}\right.$ tryptone, $5 \mathrm{gL}^{-1}$ yeast extract, $5 \mathrm{~g} \mathrm{~L}^{-1} \mathrm{NaCl}$ ) containing $100 \mathrm{mg} \mathrm{L}^{-1}$ ampicillin, $25 \mathrm{mg} \mathrm{L}^{-1}$ kanamycin and $25 \mathrm{mg} \mathrm{L}^{-1}$ chloramphenicol. $5 \mathrm{~mL}$ of overnight culture was inoculated into $1 \mathrm{~L}$ of sterile medium with antibiotics at $37^{\circ} \mathrm{C}$. When absorbance $\left(\mathrm{OD}_{600}\right)$ reached 0.7 , medium temperature was lowered to room temperature and isopropyl $\beta$-D-1-thiogalactopyranoside (IPTG) was added to the final concentration of $1 \mathrm{mmol} \mathrm{L}^{-1}$. Cells were grown at room temperature overnight in a shaking incubator.

Upon collection and lysis of H1sD2 and D2 expression cells, inclusion bodies were extensively washed in Lysis buffer $\left(50 \mathrm{mmol} \mathrm{L}^{-1}\right.$ Tris $\mathrm{pH}$ 8.0, $100 \mathrm{mmol} \mathrm{L}^{-1} \mathrm{NaCl}$ ) and afterward were solubilized in Lysis buffer with $8 \mathrm{M}$ urea, overnight at $4{ }^{\circ} \mathrm{C}$. Refolding and purification were done as in [22]. After gel electrophoresis, fractions of purified protein were pulled and applied to endotoxin removal column (Pierce ${ }^{\mathrm{TM}}$ High Capacity Endotoxin Removal Spin Columns, $0.5 \mathrm{~mL}$ ). Briefly, 4-6 mL of pulled fractions were mixed with $0,5 \mathrm{~mL}$ of endotoxin removal matrix in batch and incubated for $1 \mathrm{~h}$. Protein solution was eluted and dialyzed afterwards against PBS (phosphate buffered saline). After dialysis fractions were centrifuged, filtered and used for immunizations.

\subsection{D Page}

The homogeneity of purified recombinant D2 and H1sD2 were analyzed by 2 D PAGE according to [28]. In brief, the sample ( $25 \mu \mathrm{g}$ ) was resolved under native conditions by isoelectric focusing in $5 \%(\mathrm{w} /$ v) polyacrylamide gel containing $2.4 \%(\mathrm{v} / \mathrm{v})$ ampholytes ( $\mathrm{pH} 3.5-10$, Pharmacia). Following separation in the first dimension, the gel stripe with respective recombinant protein was incubated in equilibration buffer $\left(62.5 \mathrm{mmol} \mathrm{L}^{-1}\right.$ Tris-HCl, 5\% 2-mercaptoethanol, 2.5\% SDS, $10 \%$ glycerol) for $15 \mathrm{~min}$ and then run in the second dimension on SDSPAGE (13\%). D2 and H1sD2 were visualized following staining with Coomassie Brilliant Blue.

\subsection{CD spectroscopy}

Circular dichroism (CD) spectroscopy was used to evaluate the overall conformation of refolded D2 and H1sD2 proteins. Spectra were recorded at $25^{\circ} \mathrm{C}$ on a Jasco J-815 A spectrophotometer (Jasco, Japan). Measurements in the far-ultraviolet region $(185-260 \mathrm{~nm})$ were performed in $0.5 \mathrm{~mm}$ path length on protein samples $(0.767$ and $0.375 \mathrm{mg} \mathrm{mL}^{-1}$ for D2 and H1sD2, respectively) dialyzed overnight against a $20 \mathrm{mmol} \mathrm{L}^{-1}$ Tris buffer, $\mathrm{pH}$ 8.0. Spectra represent the average of three accumulations and were baseline-corrected by subtraction of blank buffer. Data was collected at rate of $50 \mathrm{~nm} \mathrm{~min}^{-1}$ with integration time of $4 \mathrm{~s}$.

\subsection{IgE reactivity of recombinant proteins}

IgE reactivity of recombinant D2 and H1sD2 were analyzed in immunoblot with serum from eight house dust mite allergic persons. The study was performed with the approval of the Ethics Committee of the University Children's Hospital of Belgrade, Serbia (Approval No. 017-
990/87/2012-01/19) in accordance to Serbian National guidelines (which follow the Declaration of Helsinki) for studies involving human subjects. Informed written consent was obtained prior to the study. IgE reactivity of both antigens in immunoblot was detected as previously described [22].

\subsection{Animals and immunization protocol}

In this experiment 8 week old female Balb/c mice (Military Medicine Academy, VMA, Belgrade, Serbia), were used. All experiments were approved by the Ethics Committee for the welfare of experimental animals, at the Institute of Virology, Vaccines and Sera, Torlak, Serbia, and conformed to Serbian laws and European regulations on animal welfare (Directive 2010/63/EU; Approval No. 323-07-01577/2016-05/ 13).

At days 0 and 14 mice from experimental groups $(n=6)$, were immunized by intramuscular (i.m.) injection of $2 \times 50 \mu \mathrm{L}$ of antigens in $\mathrm{PBS}$, protein amount was $12.5 \mu \mathrm{g}$ of D2 or H1sD2. Age control group received PBS only. On day 21 mice were anesthetized and bled for serum collection and on day 35 mice were terminated by cervical dislocation, and spleens and inguinal lymph nodes were collected.

\subsection{Evaluation of allergen-specific antibodies in mouse serum}

MaxiSorp ELISA plates (Nunc A/S, Denmark) were coated overnight at $4{ }^{\circ} \mathrm{C}$ with $5 \mu \mathrm{g} / \mathrm{mL}$ of purified D2 allergen in $0.1 \mathrm{M}$ sodium carbonate buffer (pH 9.6). Blocking was done with PBS $/ 2 \%$ BSA $\left(1 \mathrm{~h}, 37^{\circ} \mathrm{C}\right.$ ), the wells were filled with 100 fold diluted mice sera (in PBS containing 1\% BSA), for both IgG and IgG2a and incubated for $1 \mathrm{~h}$ at RT. Anti-mouse IgG-HRP (dilution 1:10,000, Jackson ImmunoResearch Laboratories Inc., WestGrove, PA, USA) and anti-mouse IgG2a-biotin (dilution 1:1000, Biolegend) followed by streptavidin-HRP (Biolegend) was used for detection with $o$-phenylenediamine as the substrate.

\subsection{Leukocyte extraction and flow cytometry}

Mouse spleens were removed, trimmed of excess tissue and mashed in $5 \mathrm{~mL}$ RPMI 1640 (cat no. R4130, Sigma-Aldrich) supplemented with $2 \mathrm{~g} \mathrm{~L}^{-1}$ sodium bicarbonate, $10 \mathrm{U} / \mathrm{mL}$ Penicillin/Streptomycin and $10 \%$ fetal calf serum FCS (complete RPMI). Separately mouse inguinal lymph nodes were extracted, mashed and placed in complete RPMI. Both types of cell suspensions were passed through sterile steel mesh to remove large particles and centrifuged at $400 \times g$ (Heraeus Megafuge 16R, Thermo Scientific). Pellets were resuspended and erythrocyte-depleted by incubation in $2 \mathrm{~mL}$ Lysis buffer $\left(150 \mathrm{mM} \mathrm{NH}_{4} \mathrm{Cl}, 10 \mathrm{mM} \mathrm{NaHCO}\right.$, $1 \mathrm{mM}$ disodium EDTA) for $10 \mathrm{~min}$. After centrifugation $(400 \times g$, $10 \mathrm{~min}$ ), cells were placed in complete RPMI.

For flow cytometry cell suspension was diluted in 2\% BSA in PBS with $0.1 \%$ sodium azide to $1 \times 10^{6}$ cells $/ \mathrm{mL}$. Antibodies used for cell typing were directed against $\mathrm{CD} 3 \varepsilon$ (145-2C11, Hamster IgG, FITC), CD25 (PC61.5.3, Rat IgG1, FITC), CD19 (PeCa1 Rat IgG2a PE) and CD8a (YTS 169.4, Rat IgG2b, PE), all purchased from ImmunoTools (ImmunoTools, Friesoythe, Germany). After incubation $\left(20 \mathrm{~min}, 4^{\circ} \mathrm{C}\right.$, dark) $2 \mathrm{~mL} 2 \%$ BSA in PBS with $0.1 \%$ sodium azide was added, vortexed and centrifuged ( $400 \times g$ for $10 \mathrm{~min}$ ); this step was repeated twice and the cells were finally suspended in PBS with $0,4 \%$ formaldehyde. The signal was analyzed with FACSVerse (Becton Dickinson, Mountain View, CA, USA). Lymphocyte gate was used for the estimation of population abundance.

\subsection{Cell stimulation and cytokine secretion analysis}

Spleen cells of experimental mice $\left(2 \times 10^{6} / \mathrm{mL}\right)$ were stimulated with $6 \mu \mathrm{g} / \mathrm{mL}$ of either D2 or H1sD2, for $48 \mathrm{~h}$ and supernatants were collected and stored at $-80^{\circ} \mathrm{C}$.

In supernatants, levels of IFN $\gamma$, IL4, IL5, and IL10 were measured in 
ELISA sandwich assay. Antibodies and standards were purchased from Biolegend (San Diego, CA), and were used according to the manufacturer recommendations. All washing steps included $3 \times$ washings with TPBS, and one wash with PBS. The coloured substrate was either 2,2'-azino-bis(3-ethylbenzothiazoline-6-sulphonic acid) (Sigma-Aldrich St. Louis, MO, USA) or $o$-phenylenediamine (Sigma-Aldrich, St. Louis, MO, USA). The absorbance was measured at 405 or $492 \mathrm{~nm}$ (reference wavelength $620 \mathrm{~nm}$ ).

\subsection{Statistical analysis}

Statistical analysis of cytokine and antibody levels between the experimental groups, as well as graphical presentation was performed with Origin software. Statistical analysis of lymph node and spleen lymphocyte percentages between the experimental groups, as well as graphical presentation was performed with Graph Pad software. Oneway ANOVA with Tukey test for means comparison between the groups was used. Data is expressed as mean \pm SEM. $P<0.05$ was considered significant.

\section{Results}

\subsection{Expression and isolation of $H 1 s D 2$}

To analyze immunomodulatory potential of hemagglutinin from influenza A virus in house dust mite allergy the chimera H1sD2 made of the receptor-binding domain of hemagglutinin (HA63-291, GenBank GQ149630) from influenza A (H1N1) and Der p 2 allergen (GenBank JN222809) was designed. In total 371 amino acids long, H1sD2 is 60 amino acids shorter from previously designed H1D2 chimera, lacking a part encoding for the stalk domain of H1 spike.

The H1sD2 chimera was produced in $E$. coli with $6 \mathrm{His}$ tag and after extraction from inclusion bodies and refolding; H1sD2 was purified under native conditions by $\mathrm{Co}^{2+}$ metal affinity chromatography. The chimera revealed one spot in 2D PAGE (Fig. 2A) with molecular mass of about $42 \mathrm{kDa}$ and theoretical $\mathrm{pI}$ 6.6.

Protocol for extraction of D2 from inclusion bodies and downstream purification was similar as for H1sD2. D2 revealed one spot in 2D PAGE (Fig. 2B) stained by CBB with molecular mass of $15 \mathrm{kDa}$ and theoretical $\mathrm{p} I$ 6.6.

\section{2. $C D$ spectra analysis}

Secondary structures of purified D2 and H1sD2 were analyzed by CD spectrometry (Fig. 3). CD spectra of D2 revealed minimum at about $215 \mathrm{~nm}$ and maximum at $197 \mathrm{~nm}$. The shape of CD spectra revealed $\beta$ sheeted secondary structures of D2, in agreement with the literature data [29]. $\mathrm{H} 1 \mathrm{sD} 2$ also revealed minimum at $215 \mathrm{~nm}$ which indicated presence of $\beta$-sheets.

\subsection{IgE immunoblot analysis of H1sD2 and D2}

To test IgE reactivity recombinant $\mathrm{D} 2$ and $\mathrm{H} 1 \mathrm{sD} 2$ were transferred onto a nitrocellulose membrane and were incubated with sera from eight persons with medical history of house dust mite allergy. Levels of specific IgE in patients sera to HDM allergens were as follows: class 6 ( $>100.01 \mathrm{kU}_{\mathrm{A}} / \mathrm{L}$ ) in person's no. 2, 3 and 6; class 5 (50.01-100.0 kU $\mathrm{kU}_{\mathrm{A}}$ L) in person's no. 1, 4, 7 and 8 ; and class $4\left(17.51-50.00 \mathrm{kU}_{\mathrm{A}} / \mathrm{L}\right)$ in person 5 as determined with the ImmunoCAP System (Thermo Fisher Scientific/Phadia, Uppsala, Sweden). The relative intensity of IgE reactive bands for H1sD2 and D2 were comparable (Fig. 4). The most IgE reactive sera were from person's no. 2, 3, 5, and 6 for H1sD2. Similar pattern of IgE reactivity was found for recombinant D2 allergen, except for serum no. 2 which revealed weak IgE reactivity in comparison to H1sD2. Due to the limited amount of no. 2 serum an additional analysis was not performed to clarify this discrepancy.

\subsection{Production of D2 specific antibodies}

The levels of D2 specific IgG and IgG2a were assessed with ELISA. While higher specific IgG was detected in mice injected with D2, levels of IgG2a were higher in H1sD2 injected animals (not shown). The differences between the experimental groups were not statistically significant. Nevertheless, it should be emphasized that in terms of moles of antigens employed for immunization of mice 3.4 times D2 $(1.04 \mathrm{nmol})$ was higher when compared to $\mathrm{H} 1 \mathrm{sD} 2(0.305 \mathrm{nmol})$.

\subsection{Cytokine production}

As shown in Fig. 5 the control group (PBS injected) showed basal levels of cytokines production, with no production of IL-5, or IFN- $\gamma$, and the stimulation with either antigen did not raise the level of these cytokines. The production of these cytokines were highest in D2 injected group, stimulated with $\mathrm{H} 1 \mathrm{sD} 2$ for IL-5, and the highest IFN- $\gamma$ level was produced in $\mathrm{H} 1 \mathrm{sD} 2$ injected/stimulated group, though this result was not statistically significant due to high deviation. The cells of H1sD2 injected mice also produced highest levels of IL-10, upon stimulation with H1sD2. There was no increase in production of IL-4 instead the basal level was reduced by stimulation with $\mathrm{D} 2$, and even further with H1sD2.

\subsection{Spleen and lymph node cell population analysis}

Leukocytes extracted from spleens and inguinal lymph node of mice immunized with H1sD2, D2 and PBS, respectively, were analyzed by flow cytometry. A slight increase was noted in CD19+ cell count following D2 injection in both the spleen and lymph node, but this increase was not statistically significant (Fig. 6). Concomitantly a slight decrease in $\mathrm{CD} 3$ + cell count in both compartments were found, but this also lacked statistical significance, as did the differences in CD25+ cells. Significant differences were found in the abundance of CD8+ cells, and these were location specific, such that the CD8 + cells were decreased in the lymph node of D2 immunized mice, whereas H1sD2 immunization led to an increase of CD8 + cells in both the lymph node and the spleen.

\section{Discussion}

Allergy vaccines which are based on purified or recombinant antigens are usually poorly immunogenic and require additional components to promote protective immunity. Very often adjuvants are employed to promote the immune response toward the purified or recombinant vaccines, which can reduce the number of doses required to achieve protection [30]. Viral infections, including those induced by influenza A virus provides cytokine phenotype of type 1 immunity [17], including the production of strong humoral response dominantly of IgG and IgA classes. Haemagglutinin A (HA) is the spike glycoprotein on influenza virus envelope, which binds to the receptor on host epithelial cells to invade it. As a source of immunodominant epitopes its immunogenicity has been employed in design of the first recombinant influenza vaccine FluBlok ${ }^{\circledR}$ approved by the FDA, which is composed of a trivalent recombinant hemagglutinin.

The H1sD2 chimera produced in $E$. coli was purified from inclusion bodies by $8 \mathrm{M}$ urea. After refolding the chimera revealed well defined secondary structure as well as D2 antigen, both analyzed by CD spectrometry. Computational analysis of the modeled H1sD2 chimeric structure also supports the finding that $\mathrm{H} 1 \mathrm{~s}$ and D2 domains generally retain their secondary and tertiary structure. Besides, the H1sD2 chimera showed better solubility in aqueous solutions than the previously designed H1D2 chimera, which additionally contains stalk domain decorated with high number of nonpolar amino acids. Improved solubility of the novel chimera allowed employing immunization protocol of animals without aluminium hydroxide, which per se possess a Th2- 



Fig. 2. Purified $\mathrm{D} 2$ and $\mathrm{H} 1 \mathrm{sD} 2$ were separated by isoelectric focusing according to $\mathrm{p} I$ value, and then according to molecular weight in $13 \%$ SDS PAGE and stained with CBB R250. A) 2D PAGE of H1sD2; B) 2D PAGE of D2.

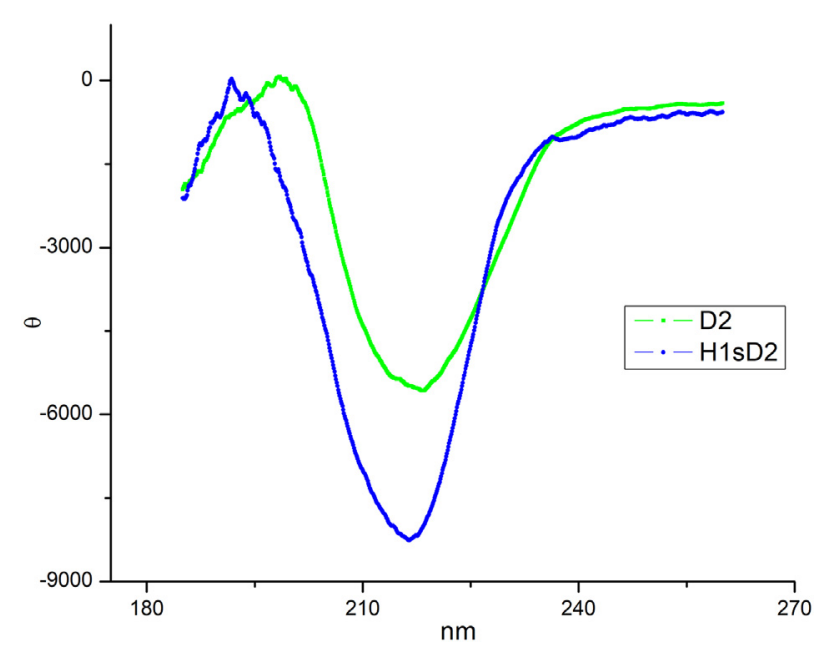

Fig. 3. CD spectra of recombinant $\mathrm{D} 2$ and $\mathrm{H} 1 \mathrm{sD} 2$ in the far-ultraviolet region $(185-260 \mathrm{~nm})$.

biasing effects [31]. Both antigens preserved IgE reactivity in immunoblot when tested with serum form house dust mite allergic persons, showing that IgE epitopes are present on the newly constructed chimera. Although western blot is not a quantitative method, the discrepancy in the intensity of IgE reactivity between D2 and H1sD2 was noticed for serum no. 2. Animal experiments were performed in order

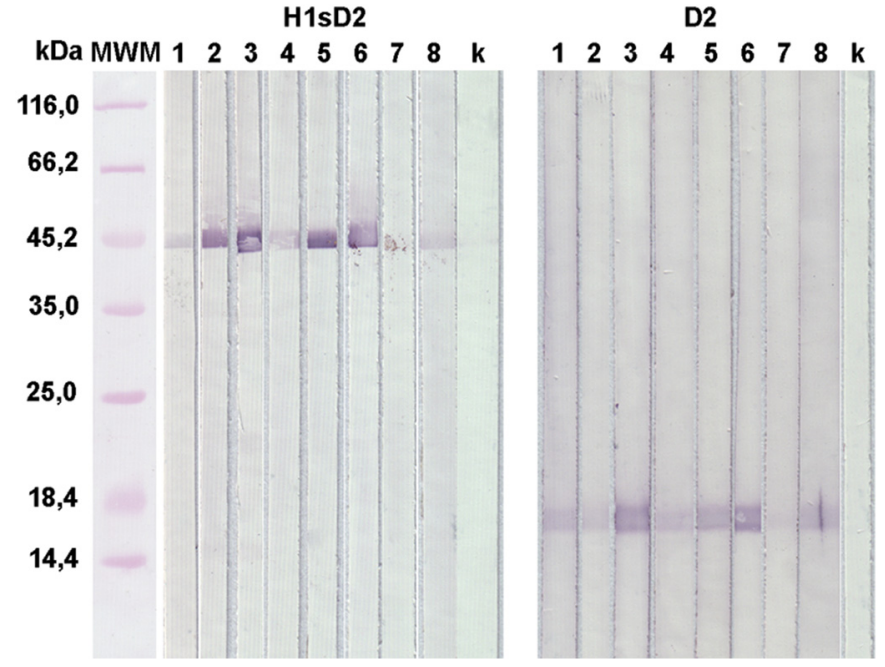

Fig. 4. IgE reactivity of sera from HDM allergic persons to H1sD2 and D2 (Nos. 1-8); $\mathrm{k}$-control of the secondary antibody (monoclonal anti-human IgE-AP). Level of HDM specific IgE in individual sera were assessed by ImmunoCAP (No. 1 - class 5; No. 2 - class 6; No. 3 - class 6; No. 4 - class 5; No. 5 - class 4, No. 6 class 6 , No. 7 - class 5 ; No. 8 - class 5 ).

to evaluate the immunomodulatory effect of the chimera H1sD2, composed of the receptor-binding domain (RBD) of hemagglutinin 1 and Der p 2 allergen, and to compare it with recombinant Der p 2 . 


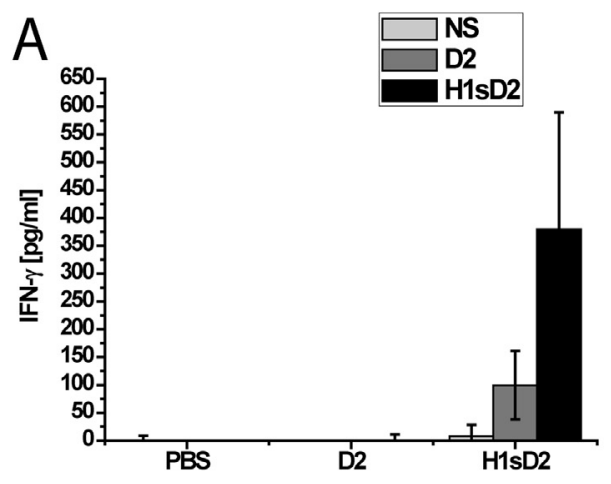



Fig. 5. Levels of different cytokines measured from splenocyte cultures supernatants, obtained from mice immunized with PBS, D2 or H1sD2, after $48 \mathrm{~h}$ of incubation with different stimulators. Samples from non-stimulated cells - NS, upon stimulation with $6 \mu \mathrm{g} / \mathrm{mL}$ of Der p2 - D2 or $6 \mu \mathrm{g} / \mathrm{mL} \mathrm{H} 1 \mathrm{sD} 2$ chimera - H1sD2. A) IFN- $\gamma$; B) IL-4; C) IL-5; D) IL-10. Mean values \pm SEM are shown, and statistical significance was assessed by ANOVA test, and $P<0.05$ is a limit of statistical significance. Statistical significance shown is obtained by comparing levels for the same stimulator between differently immunized groups.
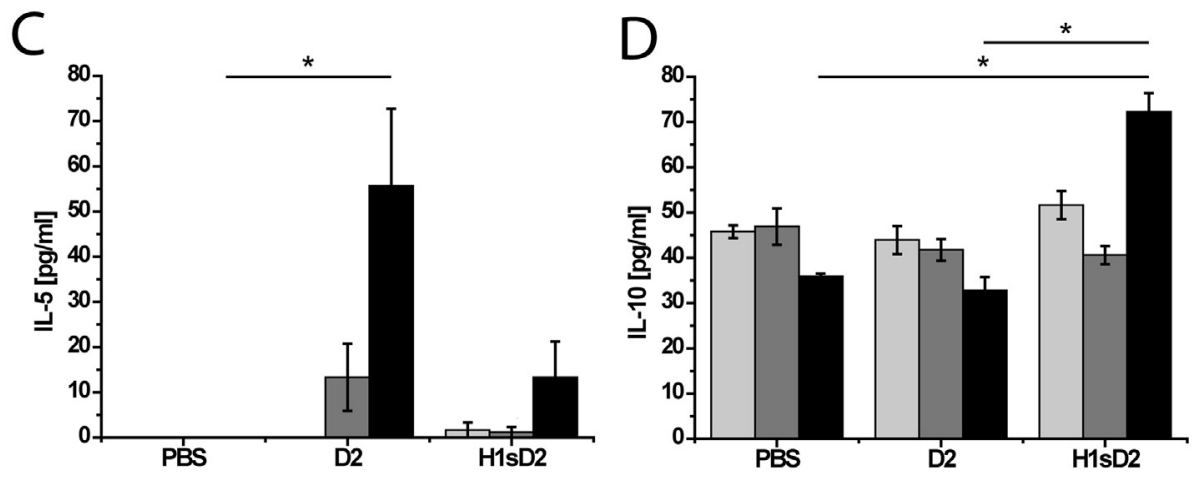

Immunization protocol was designed in such a way to assess immunogenicity, and the type of the obtained response.

Findings accumulated in the last decade shed more light on the contribution of the innate immunity in the pathogenesis of type I hypersensitivity. Epithelial-derived cytokines and chemokines also orchestrate the innate immune cells (basophils, mast cells, eosinofils, macrophages) to sustain the Th2 priming [32,33]. In the context of HDM allergy it has been clearly shown that it is not purely dys-regulation of the adaptive immunity. A tight connection between the innate and adaptive immunity is important for the initiation and propagation of the Th2 allergic response. Der p 2 has been known for some time as a trigger of the innate immune response. Its interactions with pattern-recognition receptors such as TLR2 [34] and TLR4 [16] activates distinct signaling pathways and lead to the production of inflammatory cytokines and/or type I IFNs. Here a population of splenic cells was used, which in mice consist mainly of lymphocytes (50-60\% $\mathrm{CD} 19+$ cells or B cells and $30-40 \%$ CD3 + cells or T cells) as it is convenient for assessing antigen specific responses. The differences in quantity and quality of cytokine production are known to exist between different cell types and myeloid cells, representing innate immunity, are different to lymphocytes representing adaptive immunity in this respect.

Cell stimulation experiment clearly showed that H1sD2 induces a qualitatively different type of response as compared to D2. While D2 led to an increase of IL-5 and a decrease of IL-4, H1sD2 increased IL-10, and IFN- $\gamma$, leading to a conclusion that $\mathrm{H} 1 \mathrm{sD} 2$ induced activation was more potent, and comprised of Th1 and Treg compartment activation as well. The changes found in cell population abundance, especially a significant increase in $\mathrm{CD}^{+}$cells in the spleen of H1sD2 immunized animals support the conclusion of Th1 response activation [35].

No significant differences were noted in specific antibody production upon immunization with D2 and H1sD2 antigens without adjuvant. This may be due to a relatively small number of animals used and partly due to a small number of doses. The chimera designed and produced here induces roughly the same amount of IgG in immunized animals, even though 3.4 times less moles of the chimera were used. Allergen specific immunotherapy, which is in use as an only curative approach, works by increasing protective IgG4 in humans, which is one of the parameters measured when monitoring progress of allergen specific immunotherapy [36]. In addition, influenza vaccine per se induces different kinds of protective response depending on the formulation [37]. Also, the reactivity of hemagglutinin chimeras in humans would be influenced by the preexisting immunity to the influenza virus $[38,39]$. As evidenced from the results presented here soluble chimeric antigen is able of inducing qualitatively different responses, which is seen when comparing D2 to H1sD2.

\section{Conclusion}

In this study the aqueous-soluble H1sD2 chimera was designed, produced and its immunogenicity tested in mice revealed potential for the modulation of the immune response toward Th1 and Treg phenotype. With such a chimera, without use of adjuvants, induction of Der $\mathrm{p}$ 2 -inherent Th2 immune response was attenuated in a mouse model. This finding indicates that $\mathrm{H} 1 \mathrm{sD} 2$ chimera can be an interesting candidate for application in modulating the immune response in house dust mite allergy.

\section{Acknowledgments}

This study was supported by the Ministry of Education, Science and Technological Development of the Republic of Serbia, Grant no. 172049.

\section{Author contributions}

Ivan Mrkić, Rajna Minić and Irena Živković have made substantial contributions to acquisition of data, analysis and interpretation of data; Dragan Popović have made computational analysis of chimera and has been involved in drafting the manuscript; Marija Gavrović-Jankulović has been involved in all aspects of the work in ensuring that questions related to the accuracy or integrity of any part of the work are appropriately investigated and resolved. All authors read and approved the final manuscript. 
${\mathrm{CD} 19^{+} \text {spleen }}^{-}$

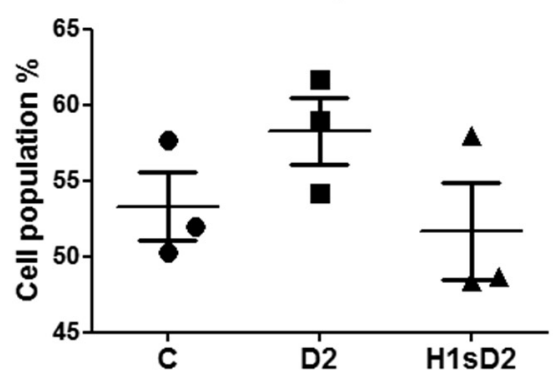

$\mathrm{CD}^{+}$spleen

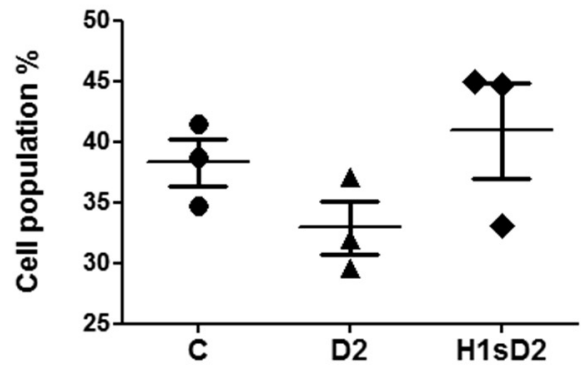

CD25 spleen



$\mathrm{CD8}^{+}$spleen

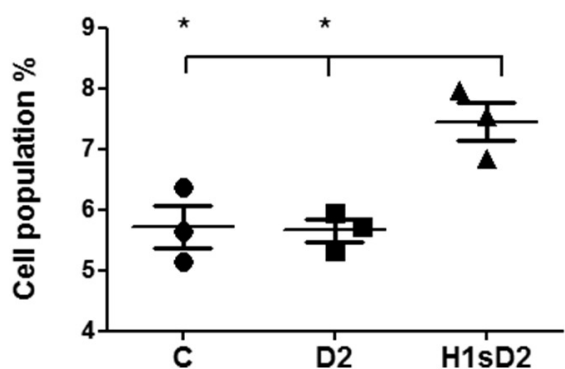

CD19+ LN
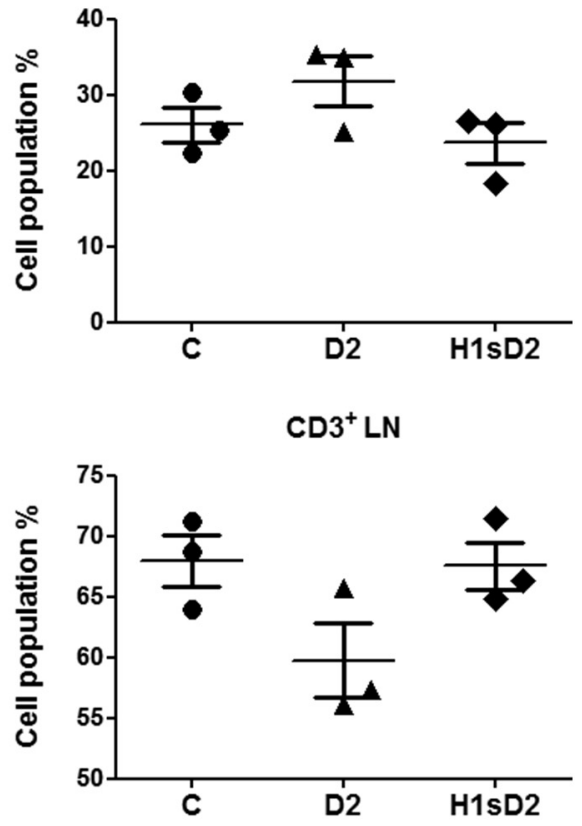

CD25 ${ }^{+}$LN


Fig. 6. Differences in spleen and lymph node lymphocyte population abundance analyzed upon day 35 from the beginning of the experiment. The data represent mean values \pm SEM from three biological replicates, each made by pooling samples from two mice. Statistical significance was determined with use of Graph Pad software and One-way ANOVA test, $P<0.05$ was considered significant. Different superscripts mark statistical significance between different groups.

\section{Conflict of interest}

The authors declare that they have no competing interests.

\section{References}

[1] R. Pawankar, Allergic diseases and asthma: a global public health concern and a call to action, World Allergy Organ. J. 7 (2014) 1-3, https://doi.org/10.1186/19394551-7-12.

[2] S.L. Prescott, Early-life environmental determinants of allergic diseases and the wider pandemic of inflammatory noncommunicable diseases, J. Allergy Clin. Immunol. 131 (2013) 23-30, https://doi.org/10.1016/j.jaci.2012.11.019.
[3] L. Noon, Prophylactic inoculation against Hay fever, Lancet 177 (1911) 1572-1573, https://doi.org/10.1016/S0140-6736(00)78276-6.

[4] S. Gilles, V. Mariani, M. Bryce, M.J. Mueller, J. Ring, T. Jakob, S. Pastore, H. Behrendt, C. Traidl-Hoffmann, Pollen-derived E1-phytoprostanes signal via PPAR-gamma and NF-kappaB-dependent mechanisms, J. Immunol. 182 (2009) 6653-6658, https://doi.org/10.4049/jimmunol.0802613.

[5] S. Gilles-Stein, I. Beck, A. Chaker, M. Bas, M. McIntyre, L. Cifuentes, A. Petersen, J. Gutermuth, C. Schmidt-Weber, H. Behrendt, C. Traidl-Hoffmann, Pollen derived low molecular compounds enhance the human allergen specific immune response in vivo, Clin. Exp. Allergy 46 (2016) 1355-1365, https://doi.org/10.1111/cea.12739.

[6] R. Valenta, R. Campana, M. Focke-Tejkl, V. Niederberger, Vaccine development for allergen-specific immunotherapy based on recombinant allergens and synthetic allergen peptides: lessons from the past and novel mechanisms of action for the future, J. Allergy Clin. Immunol. 137 (2016) 351-357, https://doi.org/10.1016/j. 
jaci.2015.12.1299

[7] M. Jutel, C.A. Akdis, Immunological mechanisms of allergen-specific immunotherapy, Allergy 66 (2011) 725-732, https://doi.org/10.1111/j.1398-9995. 2011.02589.x.

[8] M. Jutel, K. Solarewicz-Madejek, S. Smolinska, Recombinant allergens: the present and the future, Hum. Vaccin. Immunother. 8 (2012) 1534-1543, https://doi.org/ 10.4161/hv.22064.

[9] B. Linhart, M. Focke-Tejkl, M. Weber, M. Narayanan, A. Neubauer, H. Mayrhofer, K. Blatt, C. Lupinek, P. Valent, R. Valenta, Molecular evolution of hypoallergenic hybrid proteins for vaccination against grass pollen allergy, J. Immunol. 194 (2015) 4008-4018, https://doi.org/10.4049/jimmunol.1400402.

[10] P. Moingeon, Adjuvants for allergy vaccines, Hum. Vaccin. Immunother. 8 (2012) 1492-1498, https://doi.org/10.4161/hv.21688.

[11] M.A. Calderón, J. Kleine-Tebbe, A. Linneberg, F. De Blay, D. Hernandez Fernandez De Rojas, J.C. Virchow, P. Demoly, House dust mite respiratory allergy: an overview of current therapeutic strategies, J Allergy Clin Immunol Pract 3 (2015) 843-855, https://doi.org/10.1016/j.jaip.2015.06.019.

[12] M.A. Calderon, T.B. Casale, H.S. Nelson, P. Demoly, An evidence-based analysis of house dust mite allergen immunotherapy: a call for more rigorous clinical studies, J. Allergy Clin. Immunol. 132 (2013) 1322-1336, https://doi.org/10.1016/j.jaci. 2013.09.004.

[13] A.O. Eifan, M.A. Calderon, S.R. Durham, Allergen immunotherapy for house dust mite: clinical efficacy and immunological mechanisms in allergic rhinitis and asthma, Expert. Opin. Biol. Ther. 13 (2013) 1543-1556, https://doi.org/10.1517/ 14712598.2013.844226.

[14] T. Batard, V. Baron-Bodo, A. Martelet, M. Le Mignon, P. Lemoine, K. Jain, S. Mariano, S. Horiot, H. Chabre, C. Harwanegg, C.A. Marquette, B.P. Corgier, W.T. Soh, P. Satitsuksanoa, A. Jacquet, F.T. Chew, E. Nony, P. Moingeon, Patterns of IgE sensitization in house dust mite-allergic patients: implications for allergen immunotherapy, Allergy 71 (2016) 220-229, https://doi.org/10.1111/all.12796.

[15] U. Derewenda, J. Li, Z. Derewenda, Z. Dauter, G.A. Mueller, G.S. Rule, D.C. Benjamin, The crystal structure of a major dust mite allergen Der p 2, and its biological implications, J. Mol. Biol. 318 (2002) 189-197, https://doi.org/10. 1016/S0022-2836(02)00027-X.

[16] A. Trompette, S. Divanovic, A. Visintin, C. Blanchard, R.S. Hegde, R. Madan, P.S. Thorne, M. Wills-Karp, T.L. Gioannini, J.P. Weiss, C.L. Karp, Allergenicity resulting from functional mimicry of a Toll-like receptor complex protein, Nature 457 (2009) 585-588, https://doi.org/10.1038/nature07548.

[17] M. Cella, F. Facchetti, A. Lanzavecchia, M. Colonna, Plasmacytoid dendritic cells activated by influenza virus and CD40L drivea potent TH1 polarization, Nat. Immunol. 1 (2000) 305-310, https://doi.org/10.1038/79747.

[18] L. Song, V. Nakaar, U. Kavita, A. Price, J. Huleatt, J. Tang, A. Jacobs, G. Liu, Y. Huang, P. Desai, G. Maksymiuk, V. Takahashi, S. Umlauf, L. Reiserova, R. Bell, H. Li, Y. Zhang, W.F. McDonald, T.J. Powell, L. Tussey, Efficacious recombinant influenza vaccines produced by high yield bacterial expression: a solution to global pandemic and seasonal needs, PLoS One 3 (2008) e2257, , https://doi.org/10. 1371/journal.pone.0002257.

[19] R.M. Dubois, J.M. Aguilar-Yanez, G.I. Mendoza-Ochoa, Y. Oropeza-Almazan, S. Schultz-Cherry, M.M. Alvarez, S.W. White, C.J. Russell, The receptor-binding domain of influenza virus hemagglutinin produced in Escherichia coli folds into its native, immunogenic structure, J. Virol. 85 (2011) 865-872, https://doi.org/10. 1128/JVI.01412-10.

[20] N. Sriwilaijaroen, Y. Suzuki, Molecular basis of the structure and function of H1 hemagglutinin of influenza virus, Proc. Jpn. Acad. Ser. B. Phys. Biol. Sci. 88 (2012) 226-249 http://www.pubmedcentral.nih.gov/articlerender.fcgi?artid = 34101418 tool $=$ pmcentrez\&rendertype $=$ abstract, Accessed date: 3 June 2016.

[21] H.-F. Ou-Yang, X.-B. Hu, X.-Y. Ti, J.-R. Shi, S.-J. Li, H.-W. Qi, C.-G. Wu, Suppression of allergic airway inflammation in a mouse model by Der p2 recombined BCG, Immunology 128 (2009) e343-e352, https://doi.org/10.1111/j.1365-2567.2008. 02970.x.

[22] I. Mrkić, R. Minić, T. Bulat, J. Aradska, M. Atanasković-Marković, B. Drakulić,
M. Gavrović-Jankulović, Modulation of the specific immune response in Balb/c mice by intranasal application of recombinant H1D2 chimera, J. Chem. Technol. Biotechnol. 92 (2017) 1328-1335, https://doi.org/10.1002/jctb.5127.

[23] M. Hong, P.S. Lee, R.M.B. Hoffman, X. Zhu, J.C. Krause, N.S. Laursen, S.-i. Yoon, L. Song, L. Tussey, J.E. Crowe, A.B. Ward, I.A. Wilson, Antibody recognition of the pandemic H1N1 influenza virus hemagglutinin receptor binding site, J. Virol. 87 (2013) 12471-12480, https://doi.org/10.1128/JVI.01388-13.

[24] G.A. Mueller, D.C. Benjamin, G.S. Rule, Tertiary structure of the major house dust mite allergen Der p 2: sequential and structural homologies, Biochemistry 37 (1998) 12707-12714, https://doi.org/10.1021/bi980578+.

[25] I. Mrkić, R. Minić, T. Bulat, J. Aradska, M. Atanasković-Marković, B. Drakulić, M. Gavrović-Jankulović, Modulation of the specific immune response in Balb/c mice by intranasal application of recombinant H1D2 chimera, J. Chem. Technol. Biotechnol. 92 (2017) 1328-1335, https://doi.org/10.1002/jctb.5127.

[26] B.I.O.V.I.A. Dassault Systèmes, Discovery Studio Modeling Environment, Release 4, Dassault Systèmes, (2015).

[27] J.C. Phillips, R. Braun, W. Wang, J. Gumbart, E. Tajkhorshid, E. Villa, C. Chipot, R.D. Skeel, L. Kalé, K. Schulten, Scalable molecular dynamics with NAMD, J. Comput. Chem. 26 (2005) 1781-1802, https://doi.org/10.1002/jcc.20289.

[28] I. Aleksic, M. Popovic, R. Dimitrijevic, U. Andjelkovic, E. Vassilopoulou, A. Sinaniotis, M. Atanaskovic-Markovic, B. Lindner, A. Petersen, N.G. Papadopoulos, M. Gavrovic-Jankulovic, Molecular and immunological characterization of Mus a 5 allergen from banana fruit, Mol. Nutr. Food Res. 56 (2012) 446-453, https://doi.org/10.1002/mnfr.201100541.

[29] T. Takai, M. Takaoka, H. Yasueda, K. Okumura, H. Ogawa, Dilution method to refold bacterially expressed recombinant Der f 2 and Der p 2 to exhibit the secondary structure and histamine-releasing activity of natural allergens, Int. Arch. Allergy Immunol. 137 (2005) 1-8, https://doi.org/10.1159/000084607.

[30] R.L. Coffman, A. Sher, R.A. Seder, Vaccine adjuvants: putting innate immunity to work, Immunity 33 (2010) 492-503, https://doi.org/10.1016/j.immuni.2010.10. 002.

[31] H. Hogenesch, Mechanism of immunopotentiation and safety of aluminum adjuvants, Front. Immunol. 3 (2012) 406, https://doi.org/10.3389/fimmu.2012. 00406.

[32] A. Jacquet, Innate immune responses in house dust mite allergy, ISRN Allergy 2013 (2013) 735031, https://doi.org/10.1155/2013/735031.

[33] C.-F. Liu, D. Drocourt, G. Puzo, J.-Y. Wang, M. Riviere, Innate immune response of alveolar macrophage to house dust mite allergen is mediated through TLR2/-4 coactivation, PLoS One 8 (2013) e75983, , https://doi.org/10.1371/journal.pone. 0075983.

[34] Y.-L. Chiou, C.-Y. Lin, Der p2 activates airway smooth muscle cells in a TLR2/ MyD88-dependent manner to induce an inflammatory response, J. Cell. Physiol. 220 (2009) 311-318, https://doi.org/10.1002/jcp.21764.

[35] A. Srikiatkhachorn, T.J. Braciale, Virus-specific CD8 + T lymphocytes downregulate T helper cell type 2 cytokine secretion and pulmonary eosinophilia during experimental murine respiratory syncytial virus infection, J. Exp. Med. 186 (1997) 421-432 http://www.ncbi.nlm.nih.gov/pubmed/9236194 (accessed July 9, 2018).

[36] M.F. Bachmann, T.M. Kündig, Allergen-specific immunotherapy: is it vaccination against toxins after all? Allergy 72 (2017) 13-23, https://doi.org/10.1111/all. 12890.

[37] R. Petrović, B. Bufan, N. Arsenović-Ranin, I. Živković, R. Minić, K. Radojević, G. Leposavić, Mouse strain and sex as determinants of immune response to trivalent influenza vaccine, Life Sci. 207 (2018) 117-126, https://doi.org/10.1016/j.lfs 2018.05.056.

[38] A.P.D. Souza, L. Haut, A. Reyes-Sandoval, A.R. Pinto, Recombinant viruses as vaccines against viral diseases, Braz. J. Med. Biol. Res. 38 (2005) 509-522, https:// doi.org/10.1590/S0100-879X2005000400004.

[39] M. Saxena, T.T.H. Van, F.J. Baird, P.J. Coloe, P.M. Smooker, Pre-existing immunity against vaccine vectors - friend or foe? Microbiology 159 (2013) 1-11, https://doi. org/10.1099/mic.0.049601-0. 